\title{
BeppoSAX survey of Be/X-ray binary candidates
}

\author{
J. M. Torrejón ${ }^{1}$ and A. Orr ${ }^{2}$ \\ 1 University of Alicante, Department of Physics, EPS, Ap. 99, 03080 Alicante, Spain \\ 2 Astrophysics Division, Space Science Department of ESA, ESTEC, Postbus 299, 2200 AG Noordwijk, \\ The Netherlands
}

Received 8 May 2001 / Accepted 20 July 2001

\begin{abstract}
We present a BeppoSAX survey of five Be/X-ray binary candidates. We report on the identification of two of them, HD 110432 and HD 141926, as low luminosity Be/X-ray binaries. For HD 110432 we report on the detection of a pulsation period of $\sim 14 \mathrm{ks}$. Because the luminosity of these sources is low and their spectra do not require non-thermal emission models, these systems are good Be+White Dwarf candidates. If the pulsation period for HD 110432 is confirmed, this system would be the most firm Be+WD candidate found up to date. The other three objects HD 65663, HD 249179 and BD+53 2262 did not show detectable X-ray emission. We argue that, while the properties of BD+53 2262 are still consistent with a quiescent Be+Neutron Star scenario, the lack of detection for the other two objects implies that they are most probably not X-ray binaries.
\end{abstract}

Key words. stars: emission line, Be - X-rays: binaries

\section{Introduction}

During the HEAO-1 all-sky survey with the Modulation Collimator, Tuohy et al. (1988) observed several faint unidentified hard X-ray sources. These sources were associated with emission line stars located within the positional error boxes of the X-ray experiment, and consequently proposed as Be/X-ray binary candidates (BeXRBs). However, as warned by these authors, the relatively large error boxes could make some of these identifications uncertain. These sources are included in the catalogues of High Mass X-ray binaries (Liu et al. 2000). However, no observation has been reported since then with other X-ray telescopes and, consequently, their very nature as X-ray sources has not been firmly established.

BeXRBs are a major subclass of High Mass X-ray binaries (HMXRBs) where a compact object orbits a Be star in an eccentric orbit (see Apparao 1994; Coe 2000 for recent reviews). A Be star is a star of spectral type $\mathrm{B}$ and luminosity class III to $\mathrm{V}$, whose spectrum shows Balmer lines in emission as well as a strong infrared excess (Slettebak 1988). These two characteristic features seem to arise from a circumstellar envelope, whose very origin remains unclear. This envelope consists of a high density low velocity component confined at the equatorial regions and a low density high velocity wind produced mostly on the polar regions of the star (Lamers \& Waters 1987). The compact object interacts with this envelope

Send offprint requests to: J. M. Torrejón,

e-mail: jmt@disc.ua.es and accretes matter thereby producing X-rays. Most of the soft X-rays are probably absorbed in the envelope itself (Apparao 1994) and therefore the observed X-ray spectrum is usually hard, the most conspicuous emission being within the range of $2-20 \mathrm{keV}$.

The compact object is generally thought to be a neutron star $(\mathrm{Be}+\mathrm{NS})$. The X-ray luminosities usually found in BeXRBs $\left(\sim 10^{36-37} \mathrm{erg} \mathrm{s}^{-1}\right)$ and the limited range in spectral types covered by the optical counterparts of these objects (Van den Heuvel \& Rappaport 1987; Negueruela 1998) confirm this picture. Evolutionary calculations show, however, that there should be a large number of less massive binary systems with white dwarf accretors (Waters et al. 1989; Raguzova 2001). These systems would present far ultraviolet emission and could be observable as low luminosity X-ray emitters $\left(L_{\mathrm{X}} \sim 10^{32} \mathrm{erg} \mathrm{s}^{-1}\right)$. To date however, only three $\mathrm{B}+$ White Dwarf systems have been found and no Be+White Dwarf (Be+WD) system has been firmly established (see however Kurakami et al. 1986; Haberl 1995, and also Robinson \& Smith 2000, for the case of $\gamma$ Cas).

In order to assess the nature of these BeXRBs we have performed an analysis of unpublished archive data from the BeppoSAX Narrow Field Instruments for five of Tuohy's BeXRB candidates.

\section{Observations}

Results from the Low-Energy Concentrator Spectrometer (LECS; 0.1-10 keV; Parmar et al. 1997), the MediumEnergy Concentrator Spectrometer (MECS; $1.8-10 \mathrm{keV}$; 
Table 1. Our sample. $T_{\text {int }}$ is the net MECS integration time in ks. The spectral types have been taken from Liu et al. (2000). NA: not available.

\begin{tabular}{lrrlrrr}
\hline Source & RA $_{2000}$ & Dec $_{2000}$ & Sp. type & Obs. start & Obs. end & $T_{\text {int }}(\mathrm{ks})$ \\
\hline HD 249179 & 055555.05 & +284706.4 & B5ne & 19961004 05:02 & $1996100412: 10$ & 20.9 \\
HD 65663 & 075615.77 & -610558.0 & B8IIIe & $1996120409: 55$ & $1996120415: 36$ & 47.0 \\
HD 110432 & 124250.27 & -630331.0 & B0.5IIIe & $1997011411: 27$ & $1997011417: 14$ & 16.0 \\
HD 141926 & 155421.82 & -551944.8 & B2nne & $1998020203: 44$ & $1998020210: 32$ & 18.2 \\
BD+53 2262 & 193252.31 & +535245.5 & NA & $1997050415: 21$ & $1997050421: 36$ & 17.8 \\
\hline
\end{tabular}

Boella et al. 1997) and the Phoswich Detection System (PDS; $15-300 \mathrm{keV}$; Frontera et al. 1997) on-board BeppoSAX are presented. All these instruments are coaligned and referred to as Narrow Field Instruments, or NFI. The MECS consists of two (-three until 1997 May 9) grazing incidence telescopes with imaging gas scintillation proportional counters in their focal planes. The LECS uses an identical concentrator system as the MECS, but utilizes an ultra-thin entrance window and a driftless configuration to extend the low-energy response to $0.1 \mathrm{keV}$.

The non-imaging PDS consists of four independent units arranged in pairs each having a separate collimator. Each collimator was alternatively rocked on-source and $210^{\prime}$ off-source every $96 \mathrm{~s}$ during the observation.

The data from the High Pressure Gas Scintillation Proportional Counter on-board BeppoSAX (HPGSPC; 5-120 keV; Manzo et al. 1997) were not used in the present study because none of the sources were bright enough for this instrument.

Table 1 lists the BeppoSAX observation epochs and net exposure times for our sample.

Good data were selected from intervals when the elevation angle above the Earth's limb was $>4^{\circ}$ and when the instrument configurations were nominal, using the SAXDAS 2.0.0 data analysis package.

In order to produce the spectra, LECS and MECS data were extracted centered on the position of each source using radii of $4^{\prime}$ and $2^{\prime}$, respectively. These are smaller than the standard recommended radii, but they are justified in the case of faint sources. However, the light curve of HD 110432 (Fig. 1) was produced using a "full" 4' extraction radius in order to exclude any effects due to possible spacecraft "wobbling".

The PDS data are only useful in the case of our brightest source HD 110432. PDS event filtering was made using the so-called PDS "Variable Rise Time" selection method. This method increases the signal to noise ratio of the PDS data in weak sources such as ours. Background subtraction for the PDS was performed in the standard way using data obtained during intervals when the collimators were offset from the source.
Table 2. 2-10 keV fluxes measured with BeppoSAX, in units of $10^{-12} \mathrm{erg} \mathrm{s}^{-1} \mathrm{~cm}^{-2}$. The fluxes are quoted with their statistical errors at $90 \%$ confidence level.

\begin{tabular}{lc}
\hline Source & $F_{2-10}$ \\
\hline HD 249179 & $<0.19$ \\
HD 65663 & $<0.14$ \\
HD 110432 & $22.70 \pm 0.01$ \\
HD 141926 & $1.12 \pm 0.04$ \\
BD +532262 & $<0.16$ \\
\hline
\end{tabular}

Background subtraction for the imaging instruments (LECS and MECS) was performed using the appropriate standard files.

\section{Results}

The $2-10 \mathrm{keV}$ fluxes and flux upper limits for our sample are listed in Table 2 . All measured results are quoted with $90 \%$ confidence uncertainty intervals, for one parameter of interest, unless otherwise quoted.

The uncertainty on the $2-10 \mathrm{keV}$ fluxes is a combination of the statistical uncertainty on the fit (given in Table 2) and systematic effects. In the case of our relatively faint sources the systematic component is dominated by the background subtraction. By comparing different MECS and LECS background subtraction techniques, involving local and standard backgrounds, and by testing different source extraction radii, it appears that the largest systematic flux deviations are no larger than $2 \%$ of the measured fluxes.

\subsection{HD 110432 - lightcurve and spectrum}

The MECS 1.8-10 keV background-substracted barycentric corrected light curve of HD 110432, is shown in Fig. 1. The background subtraction was made using the standard composite MECS background event file. Although the resulting curve is relatively noisy, a constant X-ray flux can definitely be rejected with a confidence level greater than $99 \%$, the fit yielding a $\chi^{2}=277$ (d.o.f. $=105$, d.o.f. $=$ degrees of freedom). A linear function can equally be excluded $\left(\chi^{2}=277\right.$, d.o.f. $\left.=104\right)$. A better fit $\left(\chi^{2}=214\right.$, 
HD 110432

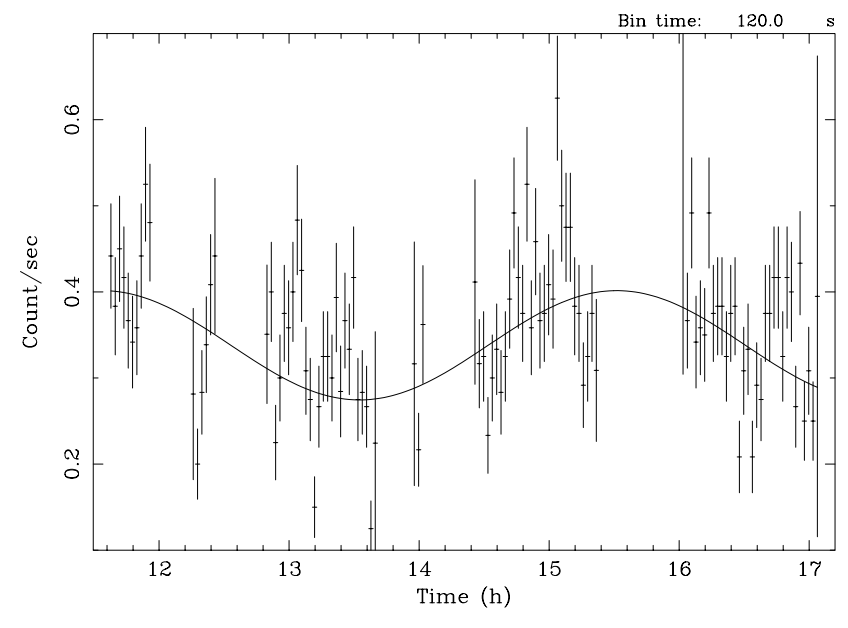

Start Time 10462 11:37:46:665 Stop Time 10462 17: 3:46:665

Fig. 1. 1.8-10 keV MECS background subtracted lightcurve of HD 110432. The data are binned in $120 \mathrm{~s}$ intervals.

Table 3. Light curve parameters for HD 110432 measured with BeppoSAX using a time binning of $120 \mathrm{~s}$.

\begin{tabular}{ccc}
\hline Parameter & Value & \\
\hline $\mathrm{A}$ & $0.34 \pm 0.01$ & $\mathrm{c} \mathrm{s}^{-1}$ \\
$\mathrm{~B}$ & $0.06 \pm 0.02$ & $\mathrm{c} \mathrm{s}^{-1}$ \\
$\phi$ & $5589 \pm 820$ & $\mathrm{~s}$ \\
$\mathrm{P}$ & $(1.42 \pm 0.14) \times 10^{4}$ & $\mathrm{~s}$ \\
\hline
\end{tabular}

d.o.f. $=102)$ is obtained with a sinusoidal curve of the type:

$A+B \sin (2 \pi(\tau-\phi) / P)$

where $A$ and $B$ are constants, $\tau$ is the time, $\phi$ is the phase and $P$ the period. The best fit parameters are presented in Table 3.

Several binning times where tried to perform the fitting. The parameters, in particular the period $P$, remained essentially unchanged. Therefore, the sinusoidal pulsation is not an artifact of the binning process. A binning time of $120 \mathrm{~s}$ was chosen in order to get a sufficient number of counts per bin to apply $\chi^{2}$ statistics while maintaining good timing resolution. Significant short time scale variations (of the order of minutes) can be seen. To see more clearly the underlying sinusoidal modulation we have tried a binning time of $2880 \mathrm{~s}$ which would sample the source twice per satellite orbit. The resulting light curve is plotted in Fig. 2. Statistically significant variations are clearly seen from bin to bin.

In order to further reject the possibility of the modulation being due to satellite wobbling a light curve analysis from a background ring around the source was performed. The result is completely compatible $\left(\chi^{2}=113\right.$, d.o.f. $=105)$ with a constant value $\left(0.26 \pm 0.01 \mathrm{cs}^{-1}\right)$. Therefore we conclude that the period must be associated with intrinsic variability of the source. The deduced

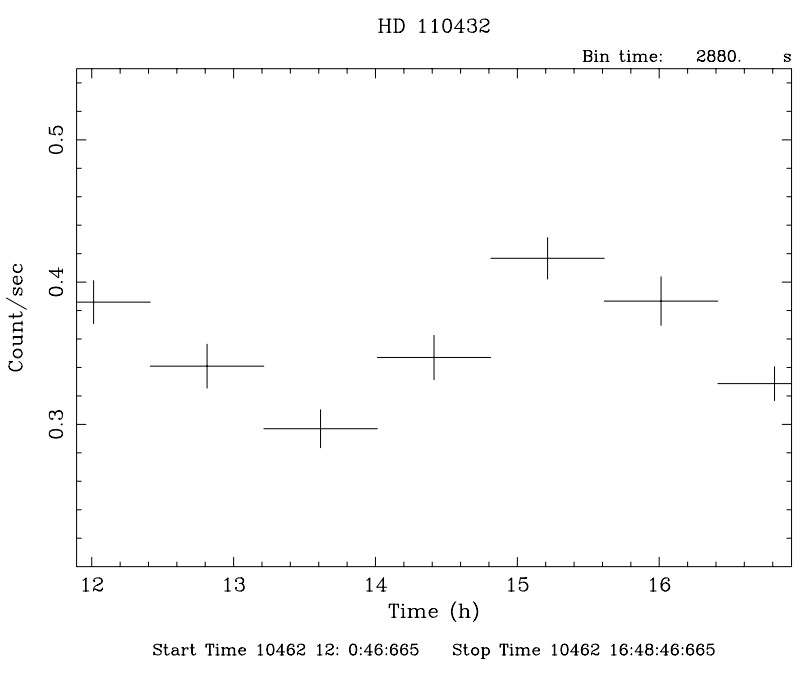

Fig. 2. The same as in Fig. 1 but with a time binning of $2880 \mathrm{~s}$. Statistically significant variations from bin to bin are clearly seen.

period ( $\sim 14 \mathrm{ks})$ is only slightly shorter than the observing window $(\sim 19 \mathrm{ks})$ so that no clear phase overlap could be established. A longer observation is clearly needed to confirm it.

The overall spectrum of HD 110432 was investigated by simultaneously fitting data from the BeppoSAX NFI, using XSPEC (Arnaud 1996). The LECS and MECS spectra were rebinned to oversample the full width half maximum of the energy resolution by a factor 3 and to have additionally a minimum of 20 counts per bin to allow use of the $\chi^{2}$ statistic. The PDS spectra were rebinned using the standard techniques in SAXDAS. Data were selected in the energy ranges $0.1-10.0 \mathrm{keV}$ (LECS), 1.65-10 keV (MECS) and 15-70 keV (PDS) where the instrument responses are well determined and sufficient counts obtained. This gives background-subtracted count rates of $0.064,0.363$, and $0.147 \mathrm{~s}^{-1}$ for the LECS, MECS and PDS, respectively.

The photoelectric absorption cross sections of Morrison \& McCammon (1983) and the solar abundances of Anders \& Grevesse (1989) are used throughout.

Factors were included in the spectral fitting to allow for normalisation differences between the instruments. These factors were constrained to be within their recommended ranges during the fitting.

Initially, simple models were tried, including absorbed power-law, and cutoff power-law models. A power-law with a photon index $\alpha=1.77 \pm 0.08$ gives an unacceptable fit with $\chi^{2}$ of 178.77 for 95 d.o.f. A cutoff power-law model with $\alpha=1.35 \pm 0.01$ and $E_{\text {cutoff }}=16.69 \mathrm{keV}$ gives a better fit with a $\chi^{2}$ of 164.17 for 94 d.o.f. Figure 3 shows the BeppoSAX spectrum of HD 110432 and the residuals to the cutoff power-law fit. Positive residuals are clearly seen around 6-7 keV and 8-9 keV, and a possible broad emission feature between $20-30 \mathrm{keV}$. The addition of Gaussian lines at 6.76 and $8.41 \mathrm{keV}$ significantly improves the fit, giving a $\chi^{2}$ of 114.89 for 91 d.o.f. for the addition of one 
HD 110432

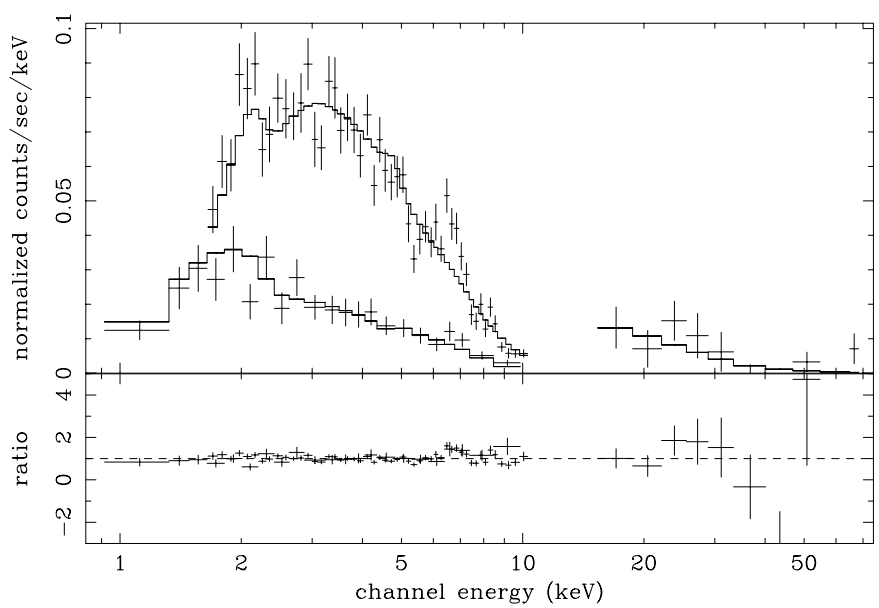

Fig. 3. LECS, MECS and PDS $0.9-50 \mathrm{keV}$ count rate spectrum of HD 110432, with data-to-model ratio in lower plot. The fitted model is a cutoff power-law with neutral photo-electric absorption. Positive residuals are clearly seen between $6-7 \mathrm{keV}$, and $7-8 \mathrm{keV}$.

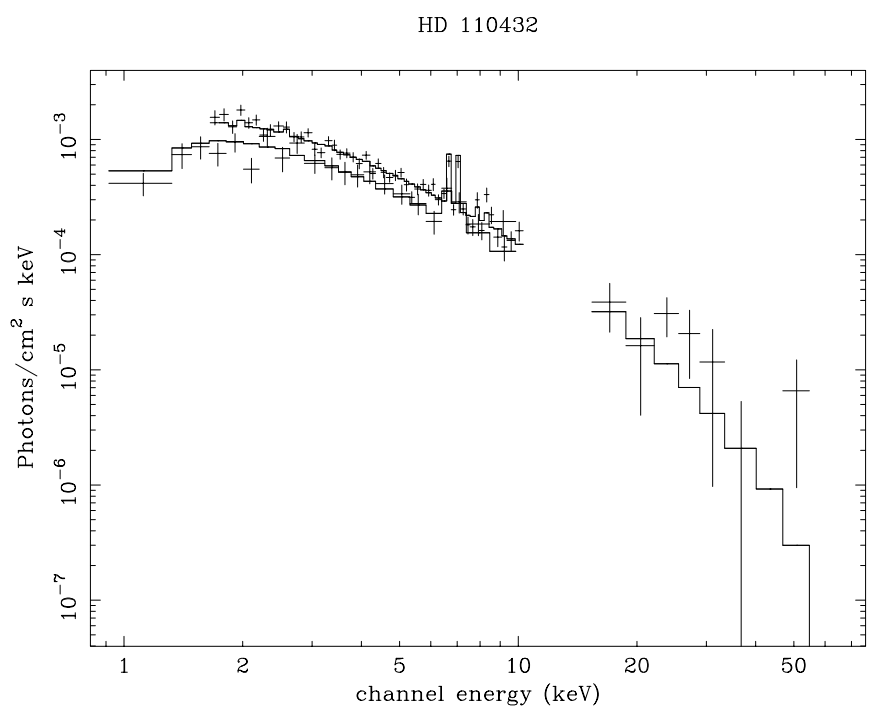

Fig. 4. The unfolded spectrum of HD 110432 with the MEKAL fitted model. Note the presence of the emission lines between 6-7 keV, and 8-9 keV.

line and $\chi^{2}$ of 107.13 for 89 d.o.f. with the addition of two lines, respectively.

A comparably good fit, however, is obtained with a MEKAL interpretation. The spectrum can be well fitted with an optically thin plasma at $k T=10.55 \mathrm{keV}\left(\chi^{2}\right.$ of 114.93 for 93 d.o.f.). The parameters for the different models are shown in Table 4 (only models with $\chi^{2} / \nu<2$ are included).

The observed $2-10 \mathrm{keV}$ flux is $F_{2-10}$ is $22.70 \times$ $10^{-12} \mathrm{erg} \mathrm{cm}^{-2} \mathrm{~s}^{-1}$ for the cutoff power-law + iron line model and $22.88 \times 10^{-12} \mathrm{erg} \mathrm{cm}^{-2} \mathrm{~s}^{-1}$ for the MEKAL model, respectively.
Table 4. Model parameters for HD 110432.

\begin{tabular}{lr}
\hline Parameter & Value \\
\hline cutoffpl & \\
& \\
$N_{\mathrm{H}}\left(10^{22} \mathrm{~cm}^{-2}\right)$ & $1.05 \pm 0.01$ \\
$\alpha$ & $1.35 \pm 0.01$ \\
$E_{\text {cutoff }}(\mathrm{keV})$ & $16.7 \pm_{9.5}^{35.6}$ \\
$\chi_{\mathrm{r}}^{2}$ (d.o.f. $)$ & $1.75(94)$
\end{tabular}

cutoffpl + one line

$\begin{array}{lr}N_{\mathrm{H}}\left(10^{22} \mathrm{~cm}^{-2}\right) & 1.25 \pm 0.30 \\ \alpha & 1.56 \pm 0.27 \\ E_{\text {cutoff }}(\mathrm{keV}) & 21.07 \pm 15.0 \\ E_{\text {line }}(\mathrm{keV}) & 6.77 \pm 0.10 \\ \sigma_{\text {line }} & 0.23 \pm 0.15 \\ E W(\mathrm{eV}) & 598 \pm 340 \\ \chi_{\mathrm{r}}^{2} \text { (d.o.f.) } & 1.26(91)\end{array}$

cutoffpl + two lines

$\begin{array}{lr}N_{\mathrm{H}}\left(10^{22} \mathrm{~cm}^{-2}\right) & 1.38 \pm 0.30 \\ \alpha & 1.63 \pm 0.27 \\ E_{\text {cutoff }}(\mathrm{keV}) & 19.9 \pm 9.5 \\ E_{\text {line1 }}(\mathrm{keV}) & 6.76 \pm 0.10 \\ \sigma_{\text {line1 }} & 0.26 \pm 0.15 \\ E W_{1}(\mathrm{eV}) & 716 \pm 430 \\ E_{\text {line2 }}(\mathrm{keV}) & 8.41 \pm 0.10 \\ \sigma_{\text {line2 }} & 0.10 \pm 0.05 \\ E W_{2}(\mathrm{eV}) & 340 \pm 204 \\ \left.\chi_{\mathrm{r}}^{2} \text { (d.o.f. }\right) & 1.20(89)\end{array}$

MEKAL

$\begin{array}{lr}N_{\mathrm{H}}\left(10^{22} \mathrm{~cm}^{-2}\right) & 1.08 \pm 0.30 \\ k T(\mathrm{keV}) & 10.55 \pm 1.90 \\ \text { Abundance } & 0.78 \pm 0.20 \\ \chi_{\mathrm{r}}^{2} \text { (d.o.f.) } & 1.24(93)\end{array}$

\subsection{HD 141926 - spectrum}

Only LECS and MECS data were used for this relatively faint source. The data were selected in the energy ranges $1.4-9.6 \mathrm{keV}$ and $1.5-12 \mathrm{keV}$, respectively, giving background-subtracted count rates of 0.004 and $0.010 \mathrm{~s}^{-1}$ for the LECS and MECS, respectively.

In order to investigate its X-ray spectrum, we initially tried simple models. Their results are listed in Table 5 . The absorbed cutoff power-law gives a very poor fit $\left(\chi^{2}=\right.$ 5.91 for 3 d.o.f.). A simple power law gives a slightly better but still poor fit ( $\chi^{2}=5.80$ for 4 d.o.f.) with photon in$\operatorname{dex} \alpha=2.12 \pm 0.02$ and an undefined absorption column with an upper limit of $2.73 \times 10^{22}$ atoms $\mathrm{cm}^{-2}$. The X-ray spectrum of HD 141926 can, however, be well described by an emission spectrum of hot, diffuse gas with a temperature of $\sim 4.5 \mathrm{keV}$ either with MEKAL interpretation $\left(k T=4.53 \mathrm{keV}, \chi^{2}=3.49\right.$ for 4 d.o.f. $)$ or a Raymond 
HD 141926

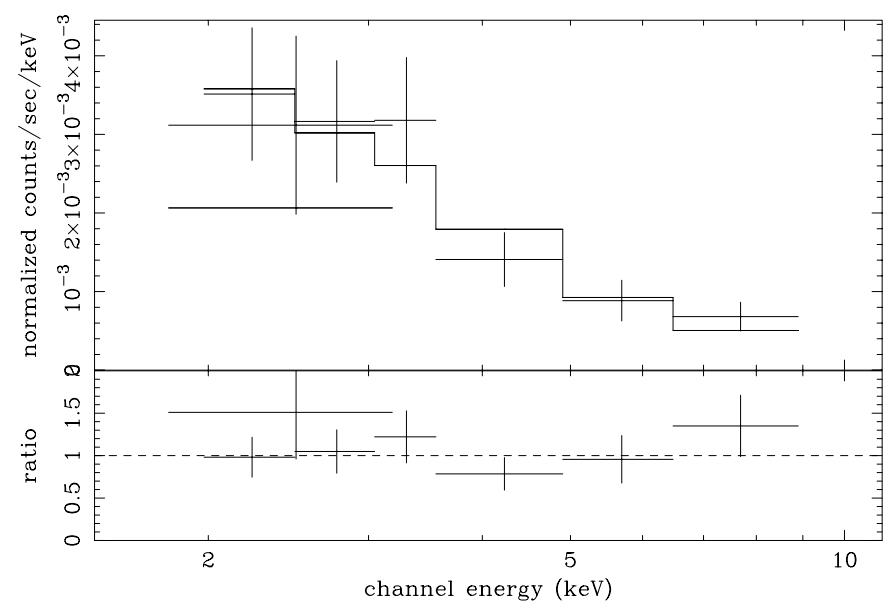

Fig. 5. LECS and MECS $2-10 \mathrm{keV}$ count rate spectrum of HD 141926, with data-to-model ratio in lower plot. The fitted model is a Raymond emission of hot ionized plasma at $k T=$ $4.08 \mathrm{keV}$ with neutral photo-electric absorption.

model interpretation $\left(k T=4.47 \mathrm{keV}, \chi^{2}=3.28\right.$ for 4 d.o.f.). The abundances in both cases have been frozen to solar. Both models yield, however, unphysically low values for the absorption column. Using additional optical data to pin down the amount of absorption, we have been able to fit rather satisfactorily the X-ray spectrum with a thermal emission of a hot, ionized, optically thin plasma model at $k T=3.9 \mathrm{keV}$. Figure 5 shows the data and fitted spectrum of HD 141926 (see Sect. 4.2 for details).

\section{3. $H D$ 249179, HD 65663 and $B D+532262$}

None of these three sources were detected during our survey. The X-ray emission, if present, was below the BeppoSAX detectable limit. Upper limits for the fluxes are given in Table 2 .

\section{Discussion}

\section{1. $H D 110432$}

This B0.5IIIe star (Codina et al. 1984) has been proposed as a possible optical counterpart of the X-ray source 1H1249-637. The unabsorbed X-ray luminosity of this object during the pointed observation was $L_{2-10 \mathrm{keV}} \simeq$ $3.4 \times 10^{32} \mathrm{erg} \mathrm{s}^{-1}$, assuming a distance of $301 \mathrm{pc}$ (ESA 1997) or $L_{2-10 \mathrm{keV}} \simeq 7 \times 10^{32} \mathrm{erg} \mathrm{s}^{-1}$ assuming a distance of $430 \mathrm{pc}$ (Codina et al. 1984). This luminosity could be generated either by coronal emission of the Be star or accretion onto the compact companion. In the first case the light curve modulation would arise from self occultation of the hot spot by the Be star. However, this leads to an unacceptably large rotation velocity at the equator. Indeed Barrera et al. (1991) found a photometric period of 1.77 days which can be regarded as the rotation period of the star (Balona 1995). Assuming a stellar radius of $\sim 15 R_{\odot}$ for a B0.5III star (Vacca et al. 1996) a rotational velocity of $430 \mathrm{~km} \mathrm{~s}^{-1}$ can be deduced. This is in agreement with the velocity deduced by Codina et al. (1984), namely $v \sin i=360 \mathrm{~km} \mathrm{~s}^{-1}$ for an inclination angle of $i=58^{\circ}$ and consistent with the absence of sharp eclipses in the light curve. This period $\left(1.77 \mathrm{~d}=15.3 \times 10^{4} \mathrm{~s}\right)$ is an order of magnitude larger than that measured from the X-ray light curve $P \sim 0.16$ d. Furthermore, the X-ray luminosity is an order of magnitude larger than that expected for a B0.5III star. The temperature deduced from the spectral fitting $(k T \sim 11 \mathrm{keV})$ is higher than that measured from coronal emission of OB stars which is typically within the range of $0.5-2.5 \mathrm{keV}$ (Pallavicini et al. 1981). This clearly rules out the coronal emission as the origin of the observed X-ray emission. On the other hand, the possibility that the light curve modulation comes from the partial occultation of the compact object during the orbital motion is clearly ruled out by Kepler's third law for the pulsation period and the radius of a B0.5III star. So we are left with partial occultation of the hot spot on the surface of the compact object during spin as a likely origin of the X-ray pulsation.

The X-ray source was first detected by the HEAO-1 experiment, with a flux $F_{2-10 \mathrm{keV}} \simeq 4.7 \times 10^{-11} \mathrm{erg} \mathrm{cm}^{-2} \mathrm{~s}^{-1}$ (Tuohy et al. 1981), while the unabsorbed flux detected by BeppoSAX is of the same order $F_{2-10 \mathrm{keV}} \simeq$ $3.2 \times 10^{-11} \mathrm{erg} \mathrm{cm}^{-2} \mathrm{~s}^{-1}$. It has not been detected by other probes due to its faintness. Indeed, it is below the threshold limit of $R X T E A S M(\sim 20 \mathrm{mCrb})$. It was observed, however, during a pointed observation with ROSAT and detected by the ROSAT All Sky Catalogue of bright sources, and identified as 1RXS J124250.1-630332. Therefore, this source is probably a persistent low luminosity source. Such an emission could be produced by a neutron star in very wide orbit accreting matter from the stellar wind. The observed X-ray luminosity is, however, an order of magnitude smaller than that usually observed in well established neutron star Be/X-ray binaries during low or quiescent states, $\sim 10^{33-34} \mathrm{erg} \mathrm{s}^{-1}$ (Negueruela 1998). The luminosity falls, however, within the predicted range $\left(10^{29-33} \mathrm{erg} \mathrm{s}^{-1}\right)$ of wind accreting white dwarfs (Waters et al. 1989).

The spectrum shows clearly an emission line at $\sim 6.8 \mathrm{keV}$ as expected from a highly ionized plasma. Owing to the large equivalent width of this line $(\sim 600-700 \mathrm{eV})$, we interpret it as an unresolved blend of the Fe XXV $(6.70 \mathrm{keV})$ and Fe XXVI $(6.97 \mathrm{keV})$ lines. This equivalent width is much larger than those found in neutron star systems $(\leq 200 \mathrm{eV})$ but it is of the order of magnitude of those found in some cataclysmic variables. The iron line at $\sim 6.4 \mathrm{keV}$ often exhibited by many neutron star binaries is not detected here while an emission feature around $\sim 8.4 \mathrm{keV}$ is observed.

The pulse period is much longer than those usually found for neutron stars, but lies within the typical values for cataclysmic variables. The spin period would imply a $P_{\text {orb }}$ of the order of $10^{2}$ days (Apparao 1994b). This period agrees with the peak of the $P_{\text {orb }}$ distribution computed by 
Raguzova (2000) for Be+WD systems. A binary system with a magnetized white dwarf in a wide orbit accreting from the stellar wind would produce persistent X-ray emission with luminosities of the order of $10^{29-33} \mathrm{erg} \mathrm{s}^{-1}$ (Waters et al. 1989) in perfect agreement with our data.

The $E(B-V)=0.40$ of the optical counterpart (Codina et al. 1984) implies an absorption of $N_{\mathrm{H}} \sim$ $0.3 \times 10^{22} \mathrm{~cm}^{-2}$ (Ryter et al. 1975). However, the absorption deduced from the X-ray data fits is much higher $\sim(1.1-1.4) \times 10^{22} \mathrm{~cm}^{-2}$. Therefore, an important amount of circumstellar material should be present near the X-ray source. This would justify the presence of strong emission lines and is consistent with the system being a Be/X-ray binary.

\section{2. $H D 141926$}

The Be star HD 141926 has been proposed as a possible optical counterpart of the X-ray source $1 \mathrm{H} 1555-552$. We have observed it in the 1.8-10 keV energy range, using BeppoSAX. The present study has allowed us to confirm, for the first time, its nature as a Be/X-ray emitting system, as was suspected from early observations with the HEAO-1 experiment.

As explained in Sect. 3.2, the non-thermal models give unsatisfactory fits to our data. Good fits are obtained with thermal emission models although the absorption column remains undefined.

To further constrain the physical parameters of the source, we have studied the optical data available in the literature. Garrison et al. (1977) quoted a B2III::npe spectral type. These authors warn that this classification should be handled with due caution since it is based on a single spectrum. Later, Reed \& Beatty (1995) quote a B2nne spectral type. The photometric colors are $(B-V)=$ 0.559 and $(U-B)=-0.465$. This allows us to compute the reddening free parameter $Q$ and use the calibration of Halbedel (1993) to deduce a spectral type of B1. We will assume a spectral type B1.5. Assuming mean values of intrinsic $(B-V)$ and $M_{V}$ as given by Schmidt-Kaler (1982) for this spectral type and luminosity classes III or $\mathrm{V}$, an interstellar reddening of $E(B-V)=0.81$ can be derived as well as distances of $\sim 610 \mathrm{pc}$ for a class $\mathrm{V}$ star or $\sim 1150$ pc for a class III star, keeping in mind that these are rough estimates. The interstellar reddening gives an absorption column of $N_{\mathrm{H}} \sim 0.55 \times 10^{22} \mathrm{~cm}^{-2}$ (Ryter et al. 1975). Since in Be type stars an extra amount of reddening is expected to come from circumstellar material, this would represent a lower limit for $N_{\mathrm{H}}$. Thus, we have constrained the absorption column in the fits by setting this lower bound. Good fits are obtained for a hot thin plasma under MEKAL or Raymond interpretations. The absorption column remains at its lower bound with an upper limit at $2.66 \times 10^{22} \mathrm{~cm}^{-2}$, implying that little circumstellar matter is present near the $\mathrm{X}$-ray source. The parameters are given in Table 5.
Table 5. Model parameters for HD 141926.

\begin{tabular}{lr}
\hline Parameter & Value \\
\hline power law & \\
$N_{\mathrm{H}}$ & $1.92 \pm 0.01$ \\
$\alpha$ & $1.45(4)$ \\
$\chi_{\mathrm{r}}^{2}$ (d.o.f.) & \\
MEKAL & \\
$N_{\mathrm{H}}\left(10^{22} \mathrm{~cm}^{-2}\right)$ & 0.55 \\
$k T(\mathrm{keV})$ & $0.97(4)$ \\
$\chi_{\mathrm{r}}^{2}($ d.o.f. $)$ & \\
Raymond & \\
$N_{\mathrm{H}}\left(10^{22} \mathrm{~cm}^{-2}\right)$ & 0.55 \\
$k T(\mathrm{keV})$ & $4.08 \pm 1.62$ \\
$\chi_{\mathrm{r}}^{2}($ d.o.f. $)$ & $0.93(4)$ \\
\hline
\end{tabular}

We can therefore describe the X-ray spectrum of HD 141926 by a hot, diffuse thin plasma at $k T \simeq 3.9 \pm$ $0.1 \mathrm{keV}$ (where the value and the error correspond to the weighted mean) and $N_{\mathrm{H}} \sim 0.55 \times 10^{22} \mathrm{~cm}^{-2}$. There is no need to invoke any of the non-thermal components characteristic of emission from accretion onto neutron stars.

The unabsorbed $2-10 \mathrm{keV}$ flux for both models is $1.3 \times 10^{-12} \mathrm{erg} \mathrm{s}^{-1} \mathrm{~cm}^{-2}$. For the distances estimated above, this would translate into a luminosity of $L_{\mathrm{X}} \simeq 5.8 \times$ $10^{31} \mathrm{erg} \mathrm{s}^{-1}$ for a class V star and $L_{\mathrm{X}} \simeq 2.1 \times 10^{32} \mathrm{erg} \mathrm{s}^{-1}$ for a class III star, respectively. These X-ray fluxes are larger than those observed in B1-B2/V-III stars due to coronal emission (Pallavicini et al. 1981) and would confirm this object as a low luminosity Be/X-ray binary. Indeed, the bolometric luminosities for a B1.5 star are $4.2 \times 10^{37} \mathrm{erg} \mathrm{s}^{-1}$ for a class V and $1.1 \times 10^{38} \mathrm{erg} \mathrm{s}^{-1}$ for a class III (Schmidt-Kaler 1982). The expected X-ray coronal emission luminosities (Pallavicini et al. 1981 ) are $L_{\mathrm{X}}=10^{-7} L_{\mathrm{bol}}=4.2 \times 10^{30} \mathrm{erg} \mathrm{s}^{-1}$ (class V) or $1.1 \times 10^{31} \mathrm{erg} \mathrm{s}^{-1}$ (class III), i.e., an order of magnitude smaller than those observed. Furthermore the measured temperature $(k T \sim 4 \mathrm{keV})$ is higher than that expected from coronal emission in early type B stars. These facts strongly suggest that HD 141926 is a low luminosity Be/X-ray binary.

\section{3. $D M+532262$}

This Be star is a proposed optical counterpart of the X-ray source $1 \mathrm{H} 1936+54$. It has never been observed by imaging $\mathrm{X}$-ray telescopes since its detection during the HEAO- 1 survey. Our observations with BeppoSAX show negative results. It is completely absent in the MECS band (2$10 \mathrm{keV}$ ) but could be at the very limit of detection in the LECS image (0.1-2 keV). The upper limit of the $0.1-2 \mathrm{keV}$ 
flux is $0.54 \times 10^{-12} \mathrm{erg} \mathrm{s}^{-1} \mathrm{~cm}^{-2}$. This lack of detection does not exclude its nature as a Be/X-ray binary because it could be in a quiescent state usually found in many $\mathrm{Be} / \mathrm{X}$-ray systems. Indeed, its optical properties, namely an early spectral type, large $\mathrm{H} \alpha$ equivalent width, and large photometric variations are compatible with this object being a long period Be/X-ray binary (Martinez \& Fabregat, priv. comm.). In such systems, the accretion of matter on to the neutron star can be halted by centrifugal inhibition which occurs when the corotation radius equals the Alfvèn radius of the neutron star magnetic field (Waters et al. 1989b).

It is worth noting that, in the MECS image, there is another source $\sim 25^{\prime}$ towards the SE, identified as $1 \mathrm{RXS}$ J193527.8+534546. This source is inside the large error box of the HEAO-1 survey of Tuohy et al. and could lead to a misidentification of DM+53 2262 as an X-ray source. As the available data (optical and X-ray) for this source is very sparse, we did not carry the analysis any further in this paper.

\subsection{HD 65663 and HD 249179}

These sources have been proposed as possible optical counterparts of the X-ray sources 1H0749-600 and $1 \mathrm{H} 0556+286$ respectively. Both are included in the catalogue of HMXRBs of Liu et al. (2000). They have not been detected during our survey and upper fluxes to their emission are listed in Table 1 . As in the previous case, these non detections do not exclude them as possible Be/X-ray binaries. However, both are late type stars. This argues against these objects being $\mathrm{Be}+$ neutron star binaries since all well established Be+NS systems have spectral types earlier than B3 in a spectral distribution which is completely different from that of isolated Be stars (Negueruela 1998). Thus, these two systems are almost certainly not neutron star accreting Be/X-ray binaries. Furthermore, it is very unlikely that these sources are Be+WD systems. Indeed, as argued by Negueruela (1998), the centrifugal inhibition of accretion would not work in these systems, so they should be persistent low luminosity sources. Owing to the lack of detection with BeppoSAX we consider it very likely that they are not X-ray binaries at all.

\section{Conclusions}

We have analyzed data for five Be/X-ray binary candidates from the list proposed by Tuohy et al. (1988). We confirm HD 110432 (=1H1249-637) and HD 141926 (=1H1555-552) as low luminosity Be/X-ray binaries. In both cases, the spectra can be well fitted by thermal emission of a hot, optically thin, plasma. Such an emission is more consistent with systems harbouring white dwarfs instead of neutron stars as the compact objects. In the case of HD 110432 we have found an X-ray pulse period of $\sim 1.42 \times 10^{4} \mathrm{~s}$ which, if confirmed by other observations, would make this object the best $\mathrm{Be}+\mathrm{WD}$ candidate found to date.

The other three objects present in our survey HD 65663, HD 249179 and BD+53 2262 did not show detectable X-ray emission. These sources where proposed as $\mathrm{Be} / \mathrm{X}$-ray binaries on the ground of positional coincidence with emission line stars within the large error boxes of HEAO-1 experiment but no detection in X-rays has been reported since. BD+53 2262 has optical characteristics which are compatible with a long orbital period $\mathrm{Be}+\mathrm{NS}$ binary. Such a system could present periods of quiescence due to the centrifugal inhibition of accretion mechanism. The other two objects in our sample are late type Be stars. They are almost certainly not Be+NS systems. Since the mechanism of centrifugal inhibition of accretion should not work in Be+WD systems, the possibility of a quiescent state is unlikely. We conclude therefore that, most probably, they are not X-ray binaries.

Acknowledgements. The BeppoSAX satellite is a joint ItalianDutch programme. JMT acknowledges the research grants GR00-182 and AYA2000-1581-C02-02. This research has made use of the SIMBAD database, CDS Strasbourg, France. We thank the referee, M. Coe, for his constructive criticism. We give credit to M. Maisack who defined the original list of sources for this BeppoSAX survey. We thank A. Parmar and the BeppoSAX LECS team for fruitful discussions.

\section{References}

Anders, E., \& Grevesse, N. 1989, Geochim. Cosmochim. Acta, 53, 197

Apparao, K. M. V. 1994, Sp. Sci. Rev., 69, 255

Apparao, K. M. V. 1994b, A\&A, 291, 775

Arnaud, K. A. 1996, in Astronomical Data Analysis Software and Systems V, ASP Conf. Ser., 101, 17

Balona, L. A. 1995, MNRAS, 277, 1547

Barrera, L. H., Mennickent, R. E., \& Vogt, N. 1991, Ap\&SS, 185,79

Boella, G., Chiappetti, L., Conti, G., et al. 1997, A\&AS, 122, 327

Codina, S. J., de Freitas Pacheco, J. A., Lopes, D. F., \& Gilra, D. 1984, A\&AS, 57, 239

Coe, M. 2000, in The Be phenomenon in early type stars, IAU Coll. 175, ASP Conf. Ser., 214, 656

Dachs, J., Engels, D., \& Jiehling, R. 1988, A\&A, 194, 167

ESA 1997, The Hipparcos and Tycho Catalogues, ESA SP-1200

Frontera, F., Costa, E., Dal Fiume, D., et al. 1997, A\&AS, 122, 371

Garrison, R. F., Hiltner, W. A., \& Schild, R. E. 1977, ApJS, 35,111

Haberl, F. 1995, A\&A, 296, 685

Halbedel, E. M. 1993, PASP, 105, 465

Lamers, H. J. G. L. M., \& Waters, L. B. F. M. 1987, A\&A, 182,20

Lang, K. R. 1991, Astrophysical Data: Planets and Stars (Springer Verlag, New York) 
Liu, Q. Z., van Paradijs, J., \& van den Heuvel, E. P. J. 2000, A\&AS, 147, 25

Manzo, G., Guarrusso, S., Santangelo, A., et al. 1997, A\&AS, 122,341

Morrison, D., \& McCammon, D. 1983, ApJ, 270, 119

Murakami, T., Koyama, K., Inque, H., \& Agraval, P. C. 1986, ApJ, 310, L31

Negueruela, I. 1998, A\&A, 338, 505

Parmar, A. N., Martin, D. D. E., Bavdaz, M., et al. 1997, A\&AS, 122, 309

Pallavicini, R., Golub, R., Vaiana, G. S., et al. 1981, ApJ, 248, 279

Raymond, J. C., \& Smith, B. W. 1977, ApJS, 35, 419

Raguzova, N. V. 2001, A\&A, 367, 848

Reed, B. C., \& Beatty, A. E. 1995, ApJS, 97, 189
Ryter, C., Cesarsky, C. J., \& Audouze, J. 1975, ApJ, 198, 103 Schmidt-Kaler, T. H. 1982, Physical Parameters of the Stars. In Landolt-Bornstein New Series, vol. 2b, ed. K. Shaifers, \& H. H. Voigt (New York, Springer Verlag)

Slettebak, A. 1988, PASP, 100, 770

Tuohy, I. R., Buckley, D. A. H., Remillard, R. A., et al. 1988, in Physics of Neutron Stars \& Black Holes (Universal Academy Press, Tokyo), 93

Van den Heuvel, E. P. J., \& Rappaport, S. 1987, in Physics of Be Stars, IAU Coll. 92, ed. A. Slettebak, \& T. P. Snow (Cambridge University Press), 291

Waters, L. B. F. M., Pols, O. R., Hogeveen, J., et al. 1989, A\&A, 220, L1

Waters, L. B. F. M., de Martino, D., Habets, G. M. H. J., \& Taylor, A. R. 1989, A\&A, 223, 207 